\title{
Linear Ultrasonic Motor for Absolute Gravimeter
}

\author{
Yue Jian ${ }^{\mathrm{a}, \mathrm{b}}$, Zhiyuan Yao ${ }^{\mathrm{a}, *}$, Vadim V Silberschmidt ${ }^{\mathrm{b}}$ \\ ${ }^{a}$ State Key Laboratory of Mechanics and Control of Mechanical Structures, Nanjing University of \\ Aeronautics and Astronautics, Nanjing 210016, China \\ ${ }^{\mathrm{b}}$ Wolfson School of Mechanical and Manufacturing Engineering, Loughborough University, \\ Loughborough LE11 3TU, UK
}

\begin{abstract}
:
Thanks to their compactness and suitability for vacuum applications, linear ultrasonic motors are considered as substitutes for classical electromagnetic motors as driving elements in absolute gravimeters. Still, their application is prevented by relatively low power output. To overcome this limitation and provide better stability,a V-type linear ultrasonic motor with a new clamping method is proposed for a gravimeter. In this paper, a mechanical model of stators with flexible clamping components issuggested, according to a design criterion for clamps oflinear ultrasonic motors. After that, an effect of tangential and normal rigidity of the clamping components on mechanical output is studied. It is followed by discussion of a new clamping method with sufficient tangential rigidity and a capability to facilitate pre-load.Additionally, a prototype of the motor with the proposed clamping method was fabricated and the performance tests in vertical direction were implemented. Experimental results show that the suggested motor has structural stability and high dynamic performance, such as no-load speed of $1.4 \mathrm{~m} / \mathrm{s}$ and maximal thrust of $43 \mathrm{~N}$, meeting the requirements for absolute gravimeters.
\end{abstract}

Key words:linear ultrasonic motor,absolute gravimeter, clamping method, design

\section{Introduction}

A precise knowledge of the magnitude of the Earth's gravitation field and its variation is crucial for various aspects of science and technology, [1-3]. It can be acquired with a high-precision absolute gravimeter and used for earthquake prediction, exploration of mineral resources and as essential reference for aircraft navigation and missile guidance.

The absolute gravimeter is regularly placed at different locations to observe the local geomagnetic field variations, so it is best to be light and portable, [4-5]. A free-fall absolute gravimeter assesses the level of gravity value by measuring the distance and time of a free fall of a body in vacuum. Such a device is designed to produce a stable and repeatable motion of a free-falling body,[6].Traditional absolute gravimeters commonly use electromagnetic motors as driving device, which can lead to complicated and heavy structures. Electromagnetic motors are complex structuresthemselves and they need also mechanical transmission components to provide rectilinear motion directly. Alinear ultrasonic motor as a replacement for an electromagnetic motoras the driving device could provide a rectilinear motion directly in the vacuum without transmission components, simplifyingthe instrument and making it 
lighter.

A linear ultrasonic motor developed in 1980s is a new type of micro motor that can be used in many fields such as aerospace and precision driving thanks to its compact structure, quick response and other advantages, [7].In 1970s, Japanese scholars were first to suggest a travelling-wave linear ultrasonic motor[8], with anexciter and a vibration absorber to produce a travelling wave in a beam to drive a slide. However, this type of motor is inefficient since the whole beam vibrated generating excessive power loss during its operation.In 1998, Japanese scholars Kurosawa et al. proposed a standing-wave linear ultrasonic motor, composed of two mutually perpendicular Langevintransducers, [9-10]. It achieved a good output performance thanks to its simple and compact structure, high driving efficiency and an obvious vibration node as a clamping point. Since then, a standing-wave linear ultrasonic motor attracted significant attentions and was used for a V-shape linear ultrasonic motor with a continuous variable cross-section designed by Yang Dong and Yao Zhiyuan in 2008 [11].This type ofa continuous variable cross-section replaced theone with the sudden changes to improve transmission efficiency of a vibration wave in the stators[12].

Thanks to their ability of working in vacuum, ultrasonic motors can be used as driving elements instead of traditional electromagnetic motors in various extreme environments [13]. In recent years, ultrasonic motors were used in Micro-lander for Mars Exploration in United States [14]. The main reasons that NASA adopted ultrasonic motors as actuators for the joint of manipulators are that the reliability of the ultrasonic motor in a vacuum is high, and they are more stable [15-16].

However, poor output performance of currentlinear ultrasonic motors limits their application in absolute gravimeters. As the driving device of an absolute gravimeter, it should always be under a measured object and avoid collisionswith a free-falling body. Itmeansthat its output acceleration must be greater than that of gravity in the process of moving down.Additionally, in the uplink process, the measured object should be lifted stably back to its original position, which needsa higher output power and more stable operation.In addition, to meet the market demands, the motor should also be simply to install and maintain, and the pre-load loading device should be calibrated and easily adjusted.

Designers of linear ultrasonic motors have always paid more attention to the structural design and a choice of vibration model. Still, a structure and an installation position of a clamping element, which is an essential connection component between ultrasonic motors and external parts, have a significant impact on output properties and efficiency of the motors.Early designs of ultrasonic motorsused a spring as its clamping elements,and the pre-load was applied to the axis of symmetry of the stator. This clamping method had a complex structure, and the gap between components was not easy to eliminate, limiting the improvement of output performance. Furthermore, the fact that the stator had only two support points also led toinstability of the motor's structure. In order to eliminate these 
negative factors, Hu was first to bringa flexible hinge (arc) clamping into the structural design of ultrasonic motors in 2011, [17]. This method included moving the clamping point from the axis of symmetry to two sides of the stator and applying pre-load by the flexible arc instead of the spring. As the number of support points increased to three, the structure of motor became more stable. However, experiments showed that the levels of normal and tangential rigidity of the flexible arc clamping were of the same order of magnitude, and low tangential rigidity constrained potential improvement in terms of output power and velocity. Additionally, the deformation of the flexible mechanism could cause a drift of the motor. To further improve the output characteristics of V-shape linear ultrasonic motorsso that it could meet technical requirements of the absolute gravimeter, a novel clamping method-single-end hinge clamping -is proposed. A mechanical model of stator with single-end hinge clamping is suggested, and it is proved that the clamping could provide high tangential rigidity with an appropriate normal rigidity. Based on this idea,a prototype was designed and manufactured and its output performance was compared with that of original motors in experiments. Subsequently, an absolute gravimeter with an ultrasonic motor as driving device was designed, effectively simplifying the structural complexity.

\section{Structural design of linearultrasonic motor for absolute gravimeter}

\subsection{Principle and structure of absolute gravimeter}

A free-fall absolute gravimeter was developed rapidly in recent decades.Currently, a typical commercial absolute gravimeter usually consists of two parts: its upper part is a falling-body containerwhile its lower part is a damping structure. Taking A10 Absolute Gravimeter produced by Micro-g LaCoste of USA as an example,in itsfalling-body container, a three-side corner cube is used as a test object. When it begins to fall freely from the top of the chamber, a light source from a stable wavelength laser deviceis split and partly reflected from the corner cube. A photosensitive diode detects the untreated fringe signal reflected parallel by the corner cube. These signals can be calibrated for the corresponding time and distance, so that the level of acceleration of the test object (i.e. the acceleration of gravity) can be obtained. A schematic of process is shown in Fig. 1. 


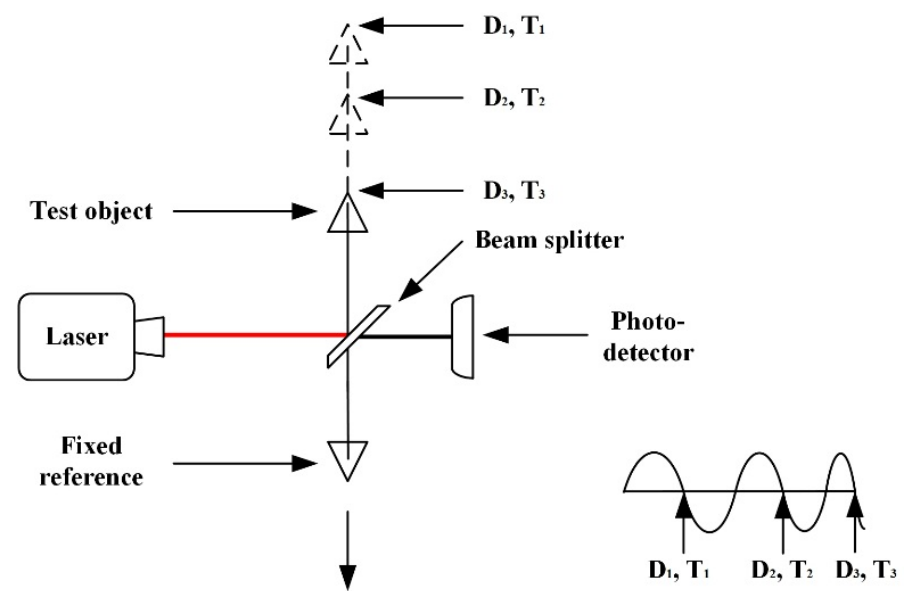

Figure 1. Measurement principle(modified after[3])

The falling-body container is a vacuum chamber, which includes the test object, the drag-free cart and mechanical transmission devices (Fig. 2). For A10 Absolute Gravimeter, a servo motor used as driving elementis installed outside of the vacuum chamber because it cannot operate in vacuum, and the drag-free cart is driven by it through a shaft.

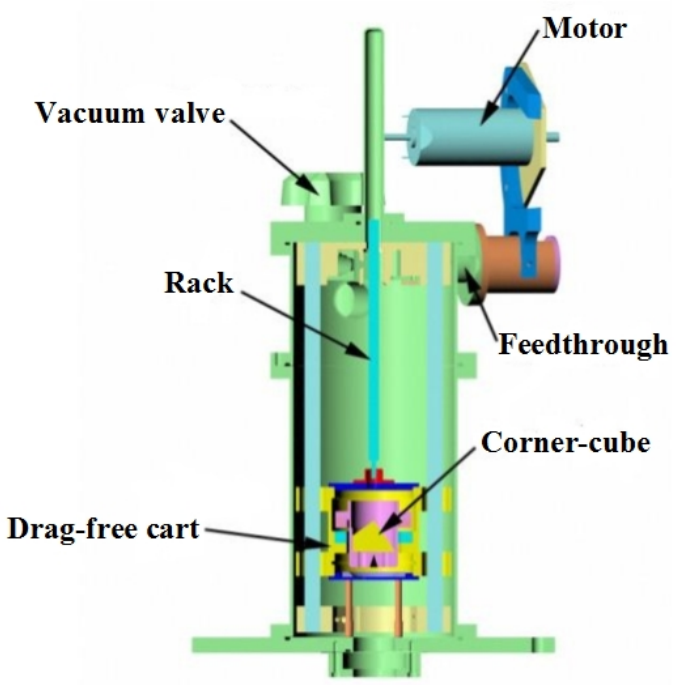

Figure 2. Schematic diagram of falling-body container

To further simplify the structure of absolute gravimeter, it is proposed to adopt ultrasonic motors instead of traditional ones as a driving element. It can be placed in the vacuum chamber and drive the drag-free cart directly. In this case,the mechanical transmission devices can be removed, reducing considerably the structural complexity and making the instrument lightweight and portable.A structure of the novel absolute gravimeter is presented in Fig.3.A stator of the ultrasonic motor is installed on the base of the vacuum chamber to drive the slide guide used as a rotor. The drag-free cart is fixed on the slide guide. When the test object falls, the drag-free cart drops with an acceleration more than $g$ at first and always maintains its position below the test object in the whole downlink process. When the test object reaches the bottom of the vacuum chamber, the drag-free cart should catch it gently and uplift it back to 

process to exceedthe weight of test object(about 20N).

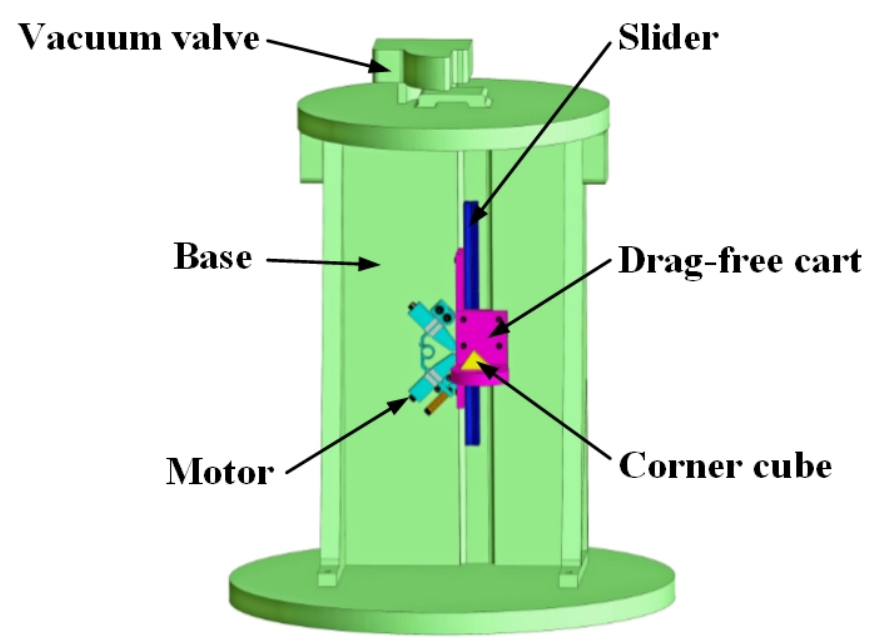

Figure 3. Structure of novel absolute gravimeter with linear ultrasonic motor

In summary, as driving element of the absolute gravimeter, the linear ultrasonic motor should reach the following technical requirements:

1. A falling distance is $50 \mathrm{~mm}$.

2. The drag-free cart maintains its position below the test object in downlink process.

3. The output thrust of the ultrasonic motor in the uplink process should be more than 20 N.

4. The ultrasonic motor can stably operate in vacuum.

5. It is easy to install, remove and maintain the motor.

\subsection{Structure design of linear ultrasonic motor for absolute gravimeter}

Existing V-shape linear ultrasonic motors could not meet the requirements of the driving element for the absolute gravimeter. In fact, their insufficient output characteristics of prevented their application in many fields [18-19]. In order to solve this problem, many researchers focused on the structural design and mode selection of the stator [20], while less attention was paid to the clamping method of the stator that also affectssignificantly the output performance of the ultrasonic motor.

As an essential component connected to external parts, the clamping element is used to support the structure of the stator, ensure flexibility of its contact with external parts. The structure and installation position of clamping has a significant effect on the output characteristics and the stability of ultrasonic motor.The existing research shows thatwith appropriate normal rigidity of the clamping element, an increase in its tangential rigidity could improve the motor's output performance[21].

According to a design criterion for clamping elements, and also taking a simple and easy-to-test method for applying pre-load into consideration, a novel clamping method of a 


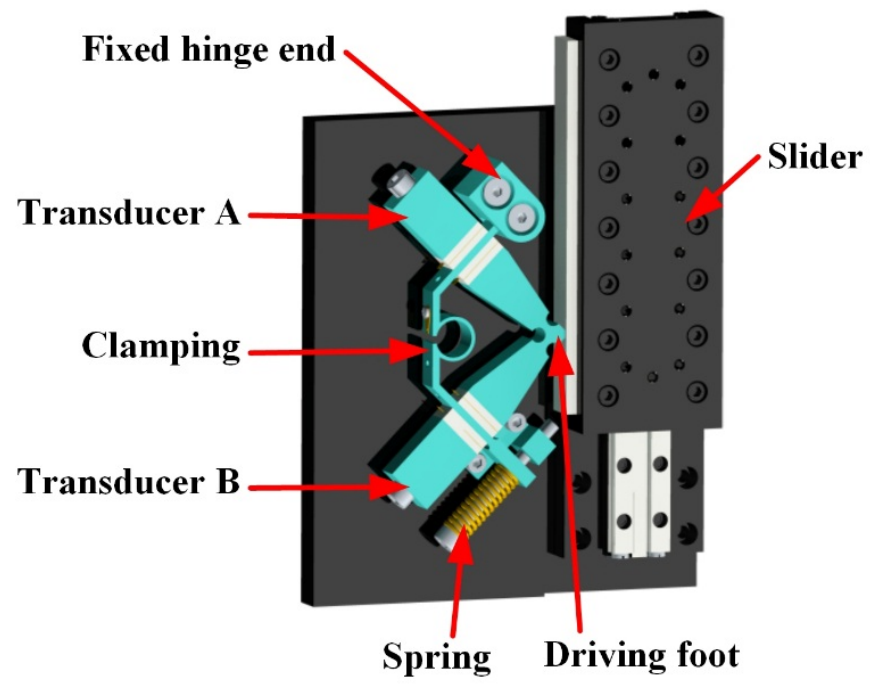

Figure 4. Overall structural design of motor

As shown in Figure 4, a stator of the V-shape ultrasonic motor is formed by two mutually perpendicular continuous variable-section Langevintransducers. When the motor is in operation, the transducers A and transducer B are excited by two sinusoidal signals with the same frequency and a $90^{\circ}$ phase difference. The vibration modes excited by these signals result in an elliptic motion of the stator's driving foot.The driving foot of the stator is pressed on the slider under a certain pre-load. In the upper half of the elliptic motion, the driving foot is in contact with the slider to drive it into linear motion. While in the lower half of elliptic motion, the slider is not in contact with the driving foot, but continues moving forward due to inertia. By repeating these sequences, the elliptic motion of the stator can be converted into the linear motion of the slider [22].An opposite motion can be achieved when reversed excitation signals are applied. It is clear from Fig.4 that the clamping element consists of a stator support, a fixed hinge end and a pre-load applying end. Stretching ofa spring can be changed by adjusting a bolt, controlling the level ofpre-load. Before the motor is operation, the pre-load between the stator and the slider can be applied by tightening the bolt to rotate the stator support around the fixed hinge end. This clamping method can improve vibration characteristics and structural stability of the motor, and increase its output power and efficiency as well. Besides, it is convenient to apply and adjust pre-load according to various occasions and requirements, thus improving controllability of the motor.

\subsection{Mechanical modal of single-hinge-end clamp}

To illustrate feasibility of the single-hinge-end clamp, its mechanical model is considered. As shown in Figure 5, connecting ahinge fulcrum, a contact point of the stator and the slider, and the point of action of pre-load loading forms a triangle rotating around thehinge fulcrum. 
When force $F$ was exerted on the pre-load loading end, pre-load $F_{n}$ would be appliedto the contact point of the stator and the slider.A distance between the point of action of the pre-load loading end and the hinge fulcrum is equal to $2 l$, and the sides of the isosceles triangle are connected at angle $\alpha$.

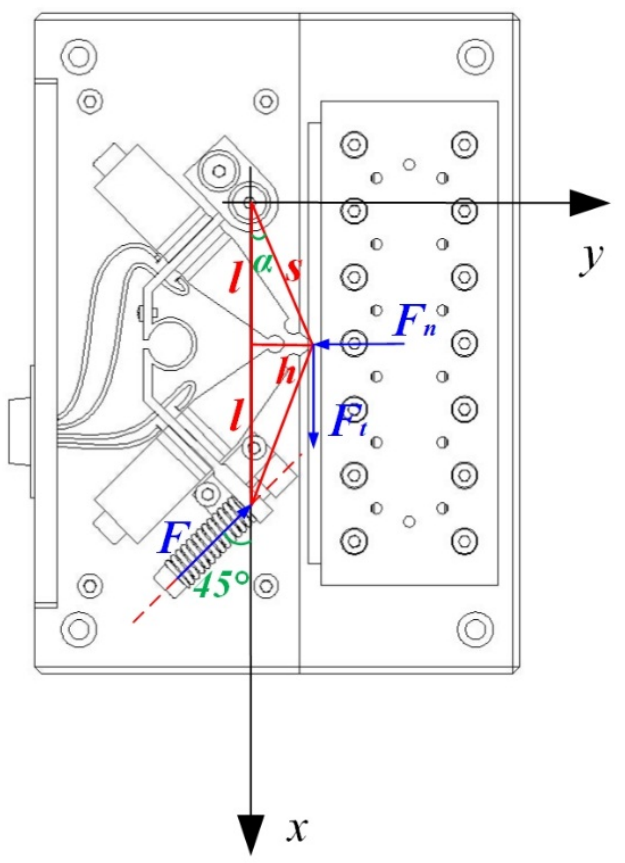

Figure 5. Mechanical model of novel clamping element

Let the value of the spring's rigidity be $K$, and assume it is placed at the angle of $45^{\circ}$ with axis $\mathrm{x}$ (Fig.5). Then its elongationof is

$$
d=\frac{F}{K} .
$$

Application of tangential force $F_{t}$ to the driving foot would producetangential displacement $S_{t}$. So, tangential rigidity of the system can be defined as

$$
K_{t}=\frac{F_{t}}{S_{t}} .
$$

From the moment balance condition

$$
F_{t} \cdot h=F \sin 45^{\circ} \cdot 2 l .
$$

At this point, the triangle would rotate around the hinge fulcrum and produce a small angle, $\Delta \alpha$. According to the relationship between displacements and employing the arc-length formula, we obtain

$$
\frac{l}{h}=\frac{s}{S_{t}} \cdot \frac{d}{\sqrt{2 l}} .
$$

Then, the relationship between tangential rigidity and that of spring can be deduced

$$
K_{t}=\frac{2 l^{3}}{\sqrt{l^{2}+h^{2}} h^{2}} K \text {. }
$$


Applying a similar procedure to a case of normal force Fn, the relationship between normal rigidity and that of spring can be obtained

$$
K_{n}=2 K \text {. }
$$

The above analysis shows that normal rigidity of the single-hinge-end clamp is constant, and two times higher than rigidity of the spring.Tangential rigidity is related to distance $h$ between the contact point of the stator and slider and the hinge fulcrum, as well as distance $2 l$ between latter and the point of action. Considering the overall size of the stator, for a given $l$, tangential rigidity increases as $h$ decreases.

\section{Assessment of output performance}

Based on the theoretical analysis above, and taking both the structure dimensions and installation requirements into account, a prototype ultrasonic motor with asingle-hinge-end clamp was designed and manufactured (see Fig.6).

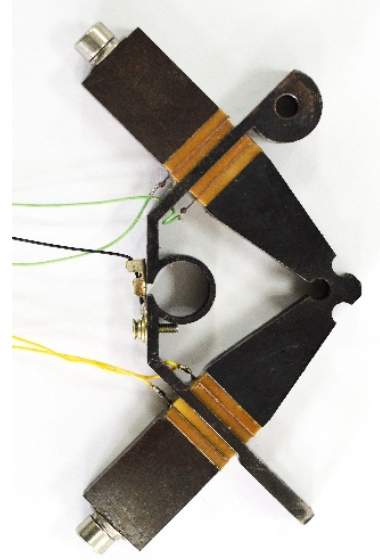

Figure 6. Prototype motor

To test the output performance of the new motor and compare it with that of the flexible-arc clamping motor (shown in Fig.7), experiments were performed with a special set up presented in Fig.8.

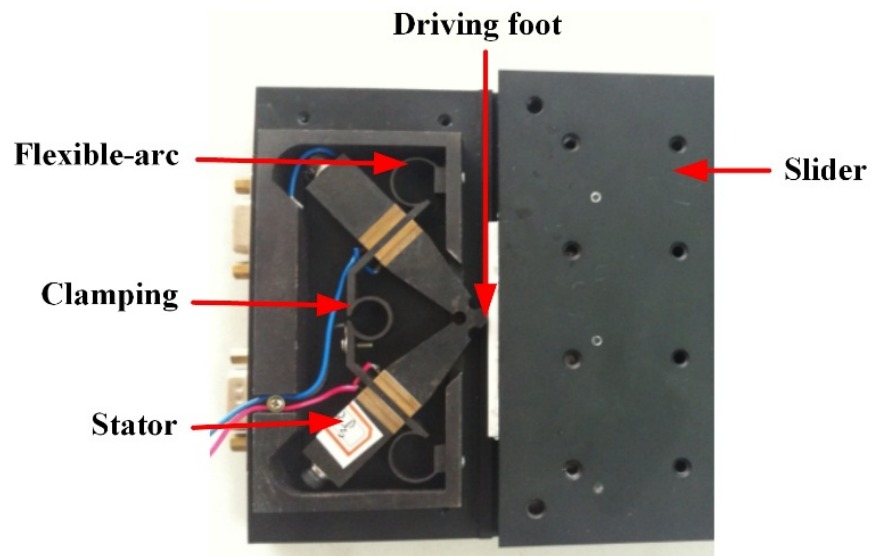

In tests with this set up, the motor was installed on the motion platform and a linear 
guide was used as aslider. When the sliderwas driven by the motor, a laser speed sensor placed on the motion platform recorded the speed of the slider and sent it to a computer. The output speed of the motor was measured for various pre-loads, driving frequencies and loads.In order to obtain the output thrust, a fixed pulley was installed on the motion platform. A rope was used to connect the sliderand the weights; the latter were considered to be equal to the output thrust. The output power wasalso measured alongside the thrust.

The effect of pre-load on output velocity and thrust of a motor with a top-top voltage of $350 \mathrm{~V}$ was studied, the respective results are presented in Figure 9.

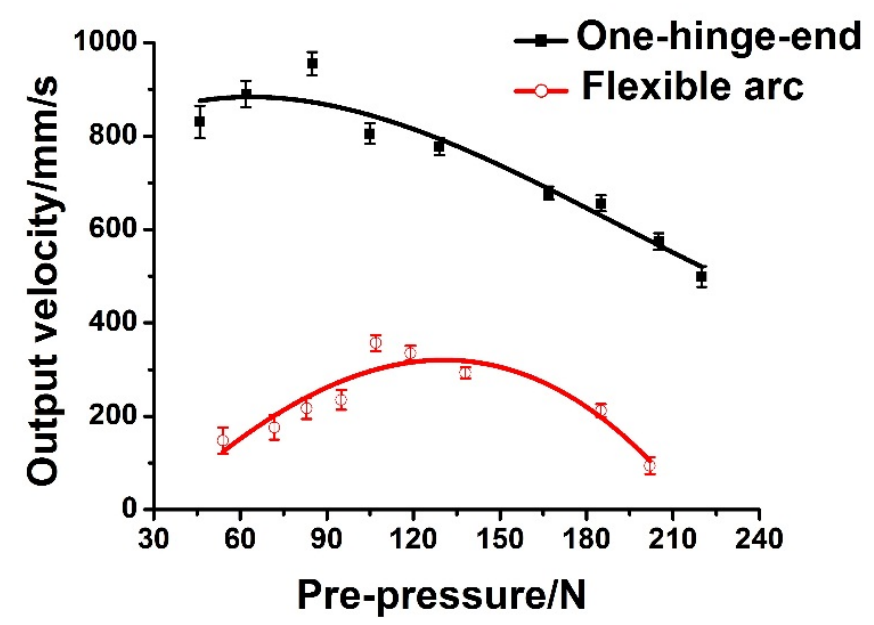




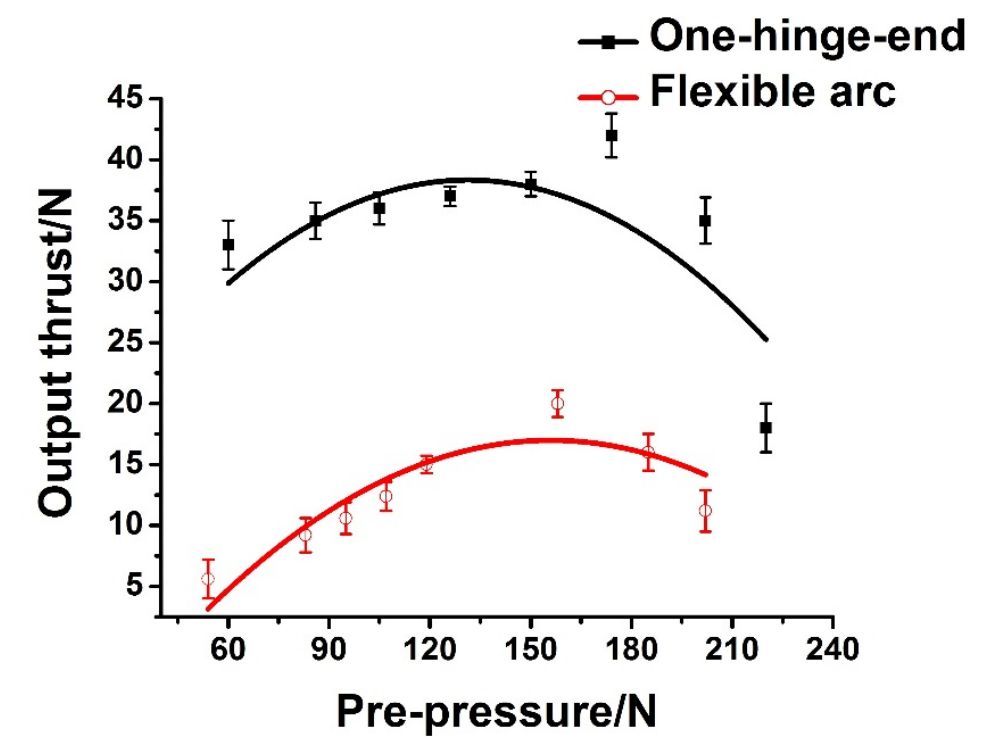

(b)

Figure 9. Effect of pre-load on output velocity (a)and thrust (b)

Apparently, with the same voltage and pre-load, the levels of outputvelocity and thrust of the novel one-end-hinge motor are higher than those of the original one. For instance, at the pre-loadof $85 \mathrm{~N}$, the no-load velocity of the proto type motor reached its maximum magnitude of $955 \mathrm{~mm} / \mathrm{s}$, nearly 2.7 times larger than that of the original motor. While the highest output thrust of $43 \mathrm{~N}$ was achieved at pre-load of $175 \mathrm{~N}$; it was 2.15 times higher than the original one. These results showed vividly that the improved design for the clamping method was efficient.

For the case ofpre-load is $85 \mathrm{~N}$, corresponding to the highest no-load velocity of the motor, the relationship between the output thrust and output velocity is presented in Fig.10.

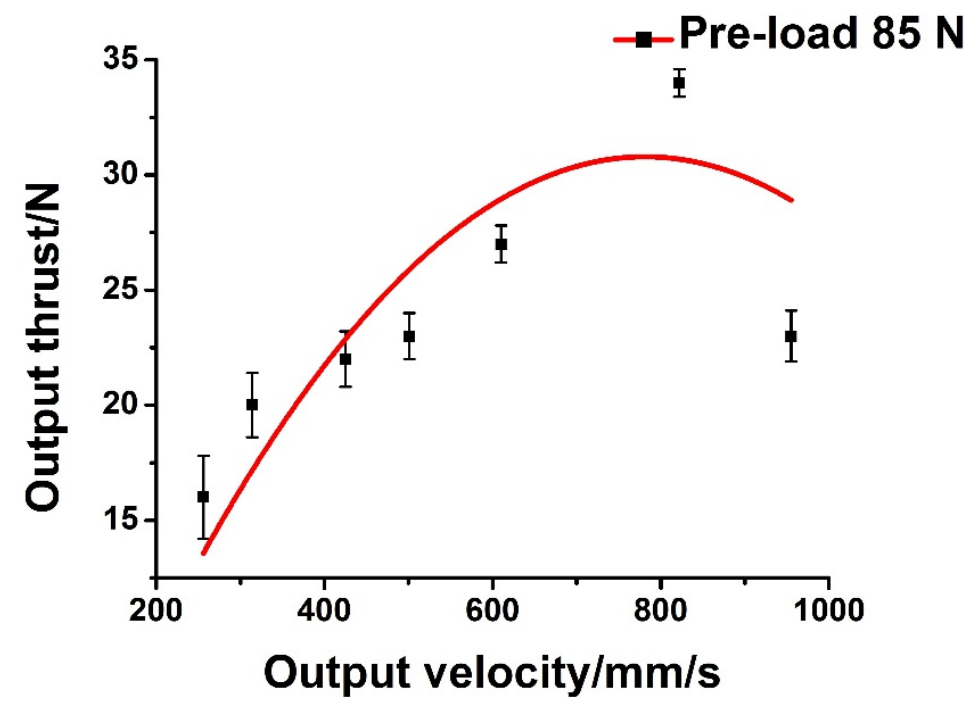

Figure 10. Relation curve between output thrust and output velocity

Maximum power of the novel motor reached the level of $20 \mathrm{~W}$ with the input voltage being $350 \mathrm{~V}$, and the corresponding current was measured to be $0.25 \mathrm{~A}$, the efficiency of motor reached $32.3 \%$. 
According to Figures9 and 10, the level of errors of output performance was smaller with a pre-load in a range between $65 \mathrm{~N}$ to $175 \mathrm{~N}$. That is because the parasiticvibration was more prominentat low pre-loads, while the usedvibration was restrained when the pre-load was too large.Hence, employingpre-loadsformthe optimal range could improve the operation stability of the motor.To confirm the output performance of the novel motor, more experiments were conducted as described below.

Asymmetry of the novel clamping method may lead to different output performances of the motor in two directions, and,as a result, affect its application.So, a comparative experiment of output velocitiesfor the two directions was conducted. The relations between the output velocity and frequencyfor two directions are shown in Figure 11 with the input voltage of $350 \mathrm{~V}$ and the pre-load of $85 \mathrm{~N}$.

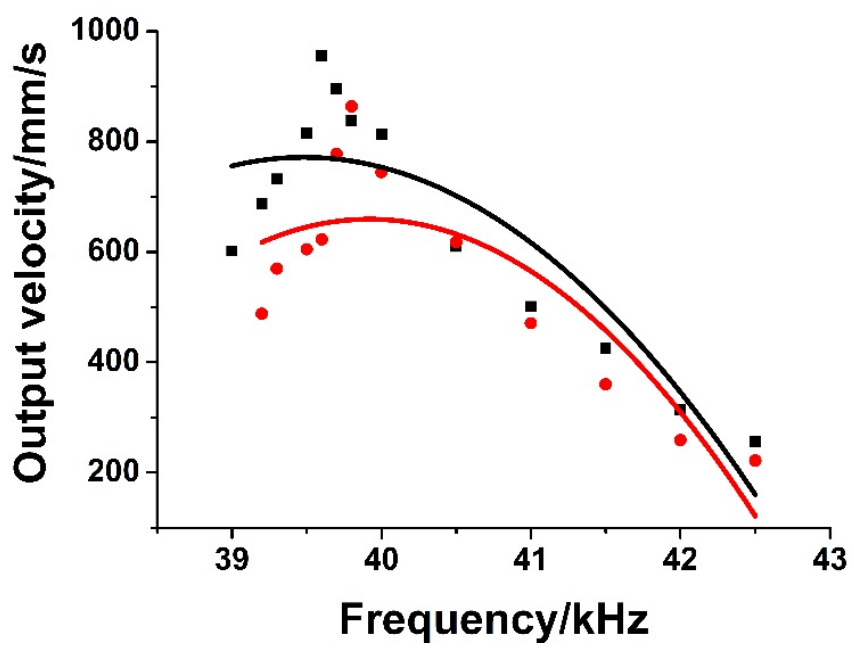

Figure 11. Relation between output velocity and frequency for two directions

According to Figure 11, asymmetry of the one-hinge-end clamping method affects the output velocities for two directions, but the difference is not considerable. Actually, there are many other factors influencing the velocity difference for these directions, e.g. roughness of contact surfaces. This directionaldifference cannot be avoided even forcompletely symmetrical motors. Still, the suggested ultrasonic motorcan operate with a high speed in both directions in a large range of frequency, hence, it can meet different requirements of operation.

For practical applications, the level of output power of the motor is not the most important technical parameter. Its stability, tunability and performanceunder loads play a more important part.

The output velocity and output thrust measured at various frequenciesfor different levels ofpre-load of 85N, 120N and 150N are shown in Figure 12. 


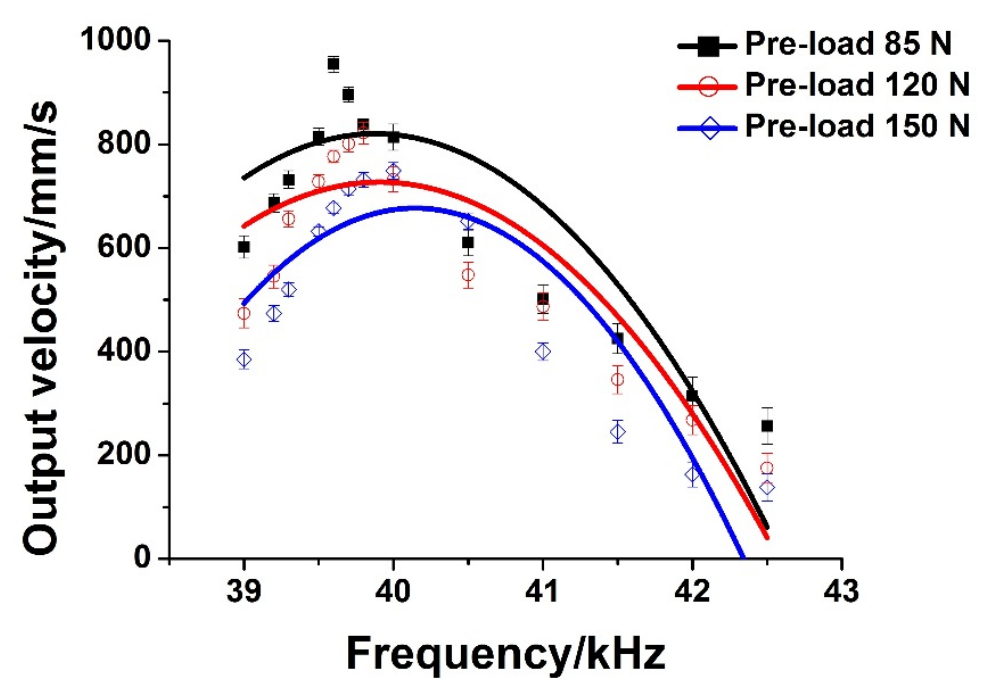

(a)

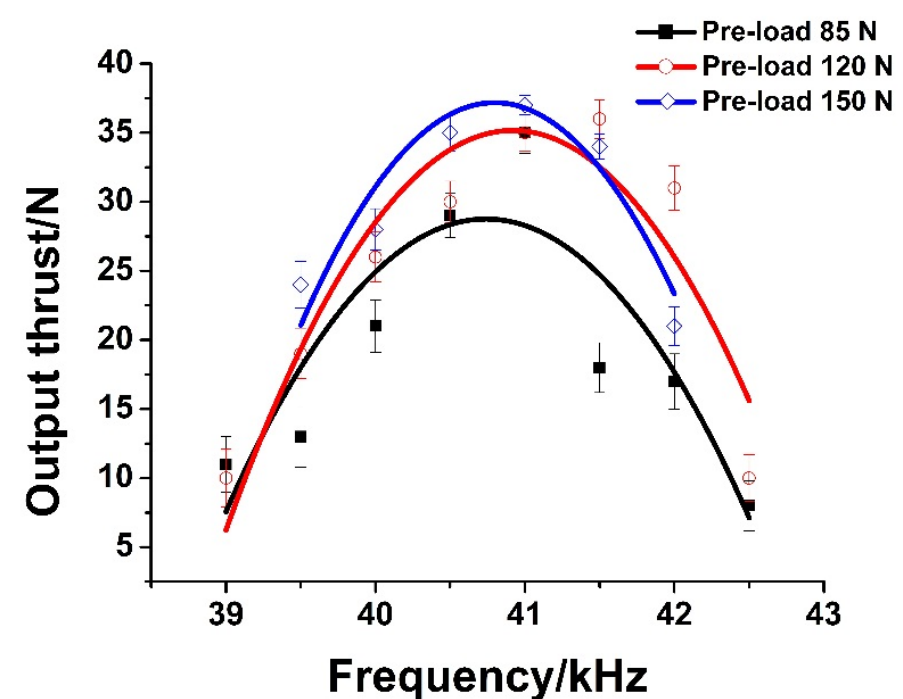

(b)

Figure 12. Effect of frequency on output velocity(a) and thrust(b) at various pre-load levels

Figure 12demonstrates that the exciting frequencies necessaryto achieve best output performanceof the motor differs somewhat at various pre-load levels. Generally, the motor can operate stably in a frequency range between 39.5 to $41.5 \mathrm{kHz}$. In summary, the range forboth exciting frequency and pre-load for the novel motor are sufficiently large. So, the novel motor can meetdifferent operation requests by fine-tuningthese two parameters for various applications.

Subsequent tests studied performance of motor under load;pre-loads of $85 \mathrm{~N}$ and loads of $10 \mathrm{~N}$ and $20 \mathrm{~N}$ were selected. The measured output velocities for various frequencies are shown in Figure 13. 


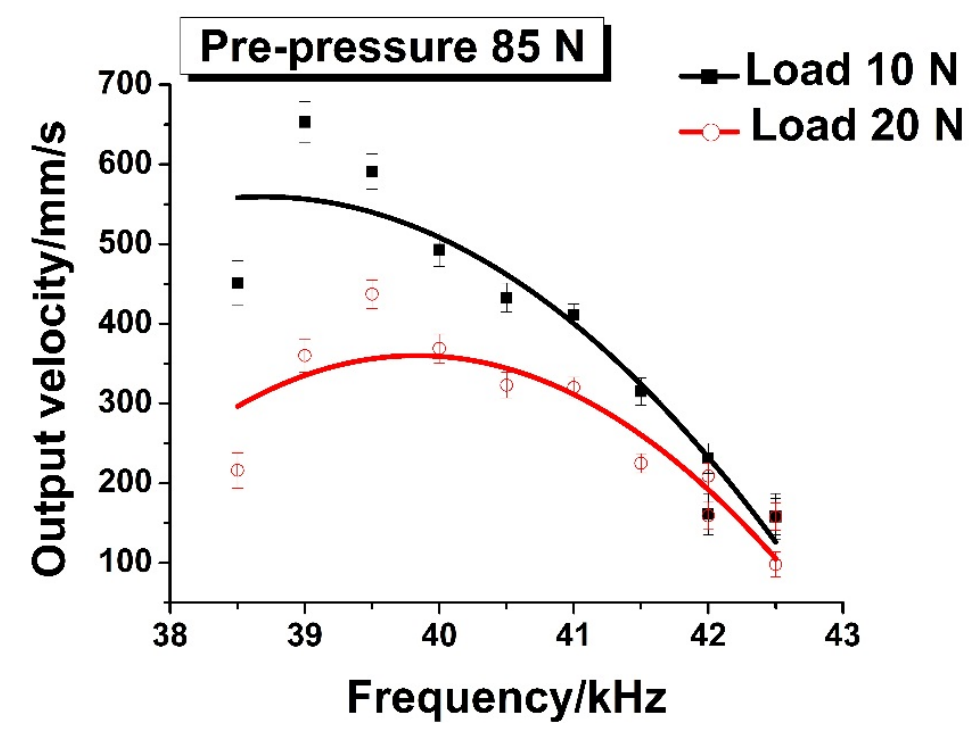

Figure13. Relation between output velocity and frequency at various loads

For the case of the $10 \mathrm{~N}$ load, the output velocity of the motor couldstably exceed 300 $\mathrm{mm} / \mathrm{s}$ within the exciting-frequency range between 39 to $41.5 \mathrm{kHz}$. Forthe exciting frequency between 39 to $40 \mathrm{kHz}$, the output velocitycould even behigherthan $500 \mathrm{~mm} / \mathrm{s}$. Although,increasing the load to $20 \mathrm{~N}$ led to the decrease of theoutput velocity, the motor couldstill operate stably in a rather large range of the exciting frequency; the small level of errors of the output velocity showed that motors operated stably. All the obtained results demonstrated that the novel motor could perform well under loads.

As the stroke of the linear guide and the measurement range of the laser speed sensor used in the tests were limited, the obtained output velocity of motor is not the maximum value. It was proved with further speed-testing experiments conducted. The motor was installed on a platform to drive a rotor, and a rotating speedometer was used to measure the motor's velocity after achieving a stable operation regime. The results showed that thehighest velocity of the motor without load was $1400 \mathrm{~mm} / \mathrm{s}$.

As the driving element of an absolute gravimeter, the ultrasonic motor will be used to provide a motion in a vertical direction. So the output performance of the motor in this direction was measured. With a falling distance of $65 \mathrm{~mm}$, input voltage $350 \mathrm{~V}$ and pre-load $85 \mathrm{~N}$, the respective velocities of the slider and the free-falling body were measured and presented in Figure 14. 


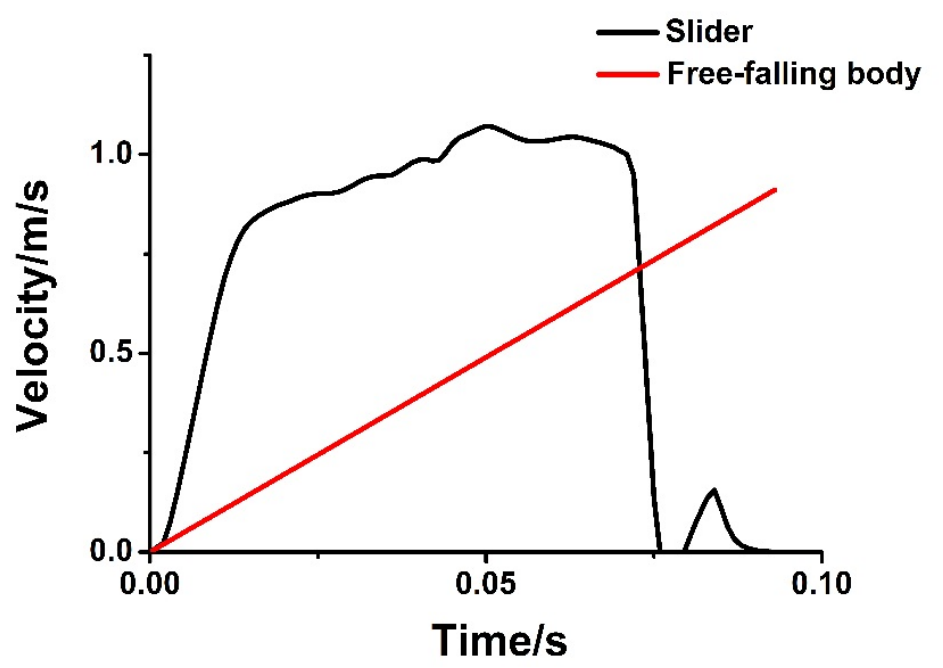

Figure14. Velocities of slider and free-falling body in downlink process

Figure 14demonstrates that when the free-falling body falls in the downlink process,under the combined effect of gravity and output thrust, the slider drops with an acceleration higher than $\mathrm{g}$ at first, hence its speed increases rapidly. The resistance increases with speed, so the slider's acceleration decreases, with its speed eventually becoming constant. But the falling distance is limited. With the slider's acceleration decreasing and the maintained acceleration of the free-falling body, the distance between them decreases. Still, in the whole downlink process,the slidercanalways maintain its position below the free-falling body. Since the motion of the slider has no effect on the fall of the free-falling body, accuracy of the measured acceleration of gravity can be guaranteed.

For the uplink process, the slider should uplift the body back to its initial position stably to conduct a next experiment. In our test, a load of $20 \mathrm{~N}$ is applied to the motor to imitate gravity of the free-falling body. The relationdisplacement-time in the uplink process is shown in Figure 15.

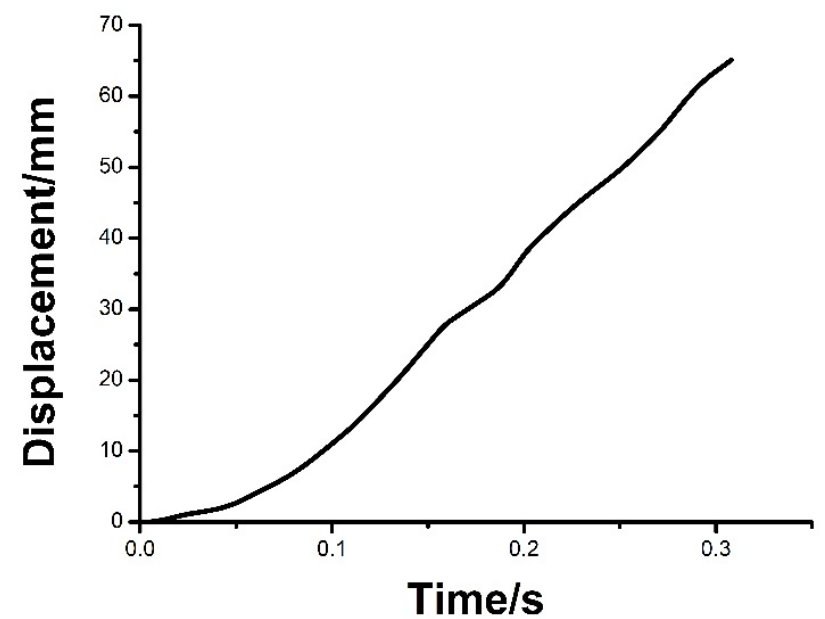


According to Figure 15, the motor can drive the slider back to its initial position with a $20 \mathrm{~N}$ load. It demonstrates that in the uplink process, the motor can overcome the gravity of the free-falling body, and uplift it stably back to its initial position for the next measurement.

In summary, compared with the original flexible-arc clamping motor, the single-hinge-end clamping motor has a better output performance, higher stability and tenability; its performance at loads and in vertical direction also improved.Thus, the novel motor can be used as the driving device of the absolute gravimeter.

\section{Conclusions}

Aiming to resolve the limitations related to free-fall absolute gravimeter, such as the complex structure and its large site, a novel absolute gravimeter based on an ultrasonic motor was proposed. In order to meet the technical requirements of the driving device for the absolute gravimeter and improve the output performance of V-shape ultrasonic motors, a novel clamping method, based on asingle-hinge-end scheme, was suggested. Its mechanical model provedtheoretically that it could meet the design criterion and improve the output performance of the motor. Considering the overall motor's dimensions and challenges of its installation, a prototype was designed and manufactured, and a series of experiments was performed to compare it with the original motor.The experiments showed that the suggestedsingle-hinge-end clamp effectively improved the output characteristics of ultrasonic motors. The achieved maximum output force was43N, and the maximum output velocity $1.4 \mathrm{~m} / \mathrm{s}$. The motor can also provide a stable motion in vertical direction. Besides,the new motor is convenient to install and adjust, and it fully meet the requirements for the driving device of theabsolute gravimeter.

\section{Acknowledgements}

This work was supported by the following funding organizations in China: the program of National Science Foundation of China (grant Number 51275229);National Key Scientific Instrument and Equipment Development Project (grant Number 2012YQ100225);China Scholarship Council; andthe Research Innovation Program forCollege Graduates of Jiangsu Province (grant Number KYLX15_0239).

\section{References}

[1] Niebauer, T. M., Sasagawa, G. S., Faller, J. E., Hilt, R., \& Klopping, F. (1995). A new generation of absolute gravimeters. Metrologia, 32(3), 159.

[2] $\mathrm{Wu}, \mathrm{Q}$. (2011). The study of the key technology in high-precision absolute gravimeter. Institute of Geophysics, China Earthquake Administration.

[3] Rothleitner, C., Svitlov, S., Mérimèche, H., Hu, H., \& Wang, L. J. (2009). Development of new free-fall absolute gravimeters. Metrologia, 46(3), 283. 
[4] Faller J E, Vitouchkine A L. (2003). Prospects for a truly portable absolute gravimeter. Journal of Geodynamics, 35(4-5):567-572.

[5] Chartier J M, Labot J, \&Sasagawa G. (1993). A portable iodine stabilized He-Ne laser and its use in an absolute gravimeter. Instrumentation \& Measurement IEEE Transactions on, 42(2):420-422.

[6] Zhao, C. (2011). Ultrasonic motors: technologies and applications. Springer Science \& Business Media.

[7] Wakai, T., Kurosawa, M. K., \& Higuchi, T. (1998). Transducer for an ultrasonic linear motor with flexible driving part. Ultrasonics Symposium. Proceedings. 683-686

[8] Kurosawa, M. K., Kodaira, O., Tsuchitoi, Y., \& Higuchi, T. (1998). Transducer for high speed and large thrust ultrasonic linear motor using two sandwich-type vibrators. Ultrasonics, Ferroelectrics, and Frequency Control, IEEE Transactions on, 45(5), 1188-1195.

[9] Yun, C. H., Ishii, T., Nakamura, K., Ueha, S., \& Akashi, K. (2001). A high power ultrasonic linear motor using a longitudinal and bending hybrid bolt-clamped Langevin type transducer. Japanese Journal of Applied Physics, 40(5S), 3773.

[10] Yang, D., \& YAO, Z. Y. (2009). Research on V-shape linear ultrasonic motor based on amplitude amplifier pole. Piezoelectrics \& Acoustooptics, 5, 022.

[11] Falk, R., Müller, J., Lux, N., Wilmes, H., \& Wziontek, H. (2012). Precise gravimetric surveys with the field absolute gravimeter A-10. In Geodesy for Planet Earth. Springer Berlin Heidelberg,273-279.

[12] Yao, Z. Y., Yang, D., \& Zhao, C. S. (2009). Structure design and power flow analysis of bar-structure linear ultrasonic Motors. Proceedings of the CSEE, 24, 010.

[13] Uchino, K. (1996). Piezoelectric actuators and ultrasonic motors (Vol. 1). Springer Science \& Business Media.

[14] Zhao, C. S. (1997). Development of micro ultrasonic motor and its prospective applications in aerospace engineering. Symposium on Application of Micro-technology for Micro-satellite. 289-295.

[15] Sherrit, S. (2005). Smart material/actuator needs in extreme environments in space. Jupiter, 1000,10000 .

[16] Alberta E F, Jiang X, \&Rehrig P (2005). Actuator materials for extreme environments. International Conference on Mems, nano and Smart Systems. IEEE Computer Society, 459-459.

[17] Hu, N., Yao, Z. Y., \& Zhao, W. T. (2012). Design of clamping components for a linear ultrasonic motor. China Mechanical Engineering, 23(9), 1089-1091.

[18] Ueha, S., Tomikawa, Y., Kurosawa, M., \& Nakamura, N. (1993). Ultrasonic motors: theory and applications (Vol. 29). Oxford University Press, USA.

[19] Uchino, K. (1998). Piezoelectric ultrasonic motors: overview. Smart Materials and Structures, 7(3), 273.

[20] Hagedorn, P., \& Wallaschek, J. (1992). Travelling wave ultrasonic motors, Part I: Working principle and mathematical modelling of the stator. Journal of Sound and Vibration, 155(1), 31-46.

[21] Jian, Y., Yao, Z. Y., Yang, M. J., \& Liu, Z. (2015). Structure design of beam type clamp for V-shape ultrasonic motor. Optics and Precision Engineering, 23(5), 1358-1364.

[22] Shi, J., Zhao, F., Shen, X., \& Wang, X. (2013). Chaotic operation and chaos control of 
travelling wave ultrasonic motor. Ultrasonics, 53(6), 1112-1123.

[23] Hou, X., Lee, H. P., Ong, C. J., \& Lim, S. P. (2013). Development and numerical characterization of a new standing wave ultrasonic motor operating in the $30-40 \mathrm{kHz}$ frequency range. Ultrasonics, 53(5), 928-934.

[24] Morita, T., Niino, T., \& Asama, H. (2002). Rotational feedthrough using ultrasonic motor for high vacuum condition. Vacuum, 65(1), 85-90.

[25] Romlay, F. R. M., Yusoff, W. A. W., \& Piah, K. A. M. (2016). Increasing the efficiency of traveling wave ultrasonic motor by modifying the stator geometry. Ultrasonics, 64, 177-185.

[26] Ho, S. T., \& Jan, S. J. (2016). A piezoelectric motor for precision positioning applications. Precision Engineering, 43, 285-293.

[27] Ri, C. S., Kim, M. J., Kim, C. S., \& Im, S. J. (2015). Study on the vibration displacement distribution of a circular ultrasonic motor stator. Ultrasonics, 59, 59-63.

[28] Lee, W. H., Kang, C. Y., Paik, D. S., Ju, B. K., \& Yoon, S. J. (2011). Butterfly-shaped ultra slim piezoelectric ultrasonic linear motor. Sensors and Actuators A: Physical, 168(1), 127-130.

[29] Leroy, E., Lozada, J., \& Hafez, M. (2014). A curved ultrasonic actuator optimized for spherical motors: Design and experiments. Ultrasonics, 54(6), 1610-1619.

[30] Jeong, S. S., Cheon, S. K., Kim, M. H., Song, J. S., \& Park, T. G. (2013). Motional characteristics of ultrasonic motor using $\Lambda$ (lambda)-shaped stator. Ceramics International, 39, S715-S719.

[31] Wan, Z., \& Hu, H. (2014). Modeling and experimental analysis of the linear ultrasonic motor with in-plane bending and longitudinal mode. Ultrasonics, 54(3), 921-928. 